\title{
Synthesis and characterization of carbon nano structures on Gallium Phosphate
}

\begin{abstract}
Carbon nano structures were grown on Gallium Phosphate substrate by using Alcohol Catalytic Chemical Vapor Deposition (ACCVD) method. The aim of this paper is to study the structure and the morphology of the carbon nano structures growth on Gallium Phosphate. Gallium Phosphate is known as piezoelectric materials which are more stable and similar to quartz in its crystal structure. The ACCVD is chosen because of its simplicity and economical method for the growth of carbon nano structure. Mixture of ethanol and Iron Nitrate in a ratio of 1:25 was used as the catalyst to impregnate the carbon nano structures. The carbon nano structures were grown at $800 \mathrm{oC}$. The ethanol liquid which was used as a carbon source was injected into the furnace tube with flow rate of $2.0 \mathrm{ml} / \mathrm{min}$. The furnace was flowed by Argon gasses throughout the experiment. FE-SEM and EDX are used to investigate the morphology of the carbon structure. Finally Raman measurements have been performed and equipped with laser diode emitting at $632 \mathrm{~nm}$.
\end{abstract}

Keyword: Carbon nanostructures; Gallium Phosphate; Raman spectrum 